\title{
Introducción a la simulación clínica
}

\author{
Ariana Cerón-Apipilhuasco ${ }^{1 *}$, Laura Rodríguez-Cruz ${ }^{1}$, Mario T. Mendoza-Carrasco ${ }^{2}$ y \\ Jorge Loria-Castellanos ${ }^{3}$ \\ ${ }^{1}$ Centro de Simulación para la Excelencia Clínica y Quirúrgica, Centro Médico Nacional Siglo XXI, Instituto Mexicano del Seguro Social (IMSS); \\ ${ }^{2}$ Unidad de Medicina Familiar 19, IMSS; ${ }^{3}$ División de Proyectos Especiales en Salud, IMSS. Ciudad de México, México
}

\section{Resumen}

Actualmente son muchos los instrumentos que han surgido de los procesos de reforma en la seguridad y calidad en salud, y algunos conceptos, como la reingeniería de los sistemas de salud, comienzan a adquirir reconocimiento. La simulación surge como una metodología de entrenamiento que facilita y ayuda a superar los procesos de cambio para las personas $y$ los equipos de trabajo. La simulación cumple con el proceso de aprender mediante la experiencia, el cual consiste en tener metas, practicar, reflexionar y conceptualizar, crear conceptos mediante los cuales integrar las observaciones en teorías lógicas, que se utilizan para la toma de decisiones y la resolución de problemas.

Palabras clave: Simulación clínica. Educación médica. Habilidades. Destrezas.

\section{Introduction to clinical simulation}

\begin{abstract}
Currently, many instruments have emerged in the processes of health security and quality reform, and some concepts, as the reengineering of health systems. The simulation as a training methodology that helps to overcome and facilitates change processes for people and work teams. The simulation complies with the process of learning through experience, which consists of having goals, practicing, reflecting, and conceptualizing, creating concepts through which they integrate their observations into logical theories, which require decision-making to solve problems.
\end{abstract}

Key words: Clinical simulation. Medical education. Skills. Proficiency.

\section{Introducción}

Uno de los objetivos de la simulación es preparar y habilitar a los estudiantes en la simulación de los eventos adversos. Permite a los estudiantes desarrollar habilidades sociales necesarias para afrontar con mayor seguridad una situación real en el contexto de su práctica profesional, así como brindar seguridad en la atención al paciente, realizar una práctica reflexiva, el desarrollo de competencias y la integración de la teoría con la práctica'.

\section{Correspondencia:}

*Ariana Cerón-Apipilhuasco

E-mail: ariana.ceron85@gmail.com
Disponible en internet: 03-12-2020 Rev Educ Investig Emer. 2019;1(4):140-144 www.medicinadeemergencias.com 2604-6520 @ 2020 Sociedad Mexicana de Medicina de Emergencias, AC. Publicado por Permanyer México SA de CV. Este es un artículo open access bajo la licencia CC BY-NC-ND (http://creativecommons.org/licenses/by-nc-nd/4.0/). 


\section{Historia de la simulación clínica}

La simulación ha existido durante siglos en muchas áreas. Los simuladores en el área de la salud se remontan a la Francia del s. xviii, cuando Madame Du Coudray usó un modelo fetal y de la pelvis para capacitar a las parteras. El movimiento moderno en el entrenamiento de simulación coincidió con el desarrollo de simuladores de tareas parciales².

Entre 1940 y 1990 en la aviación entre un 65-70\% de los accidentes que se registraban eran atribuidos a errores humanos, motivo por el cual se buscó una estrategia para reducirlos, lo que dio como resultado el uso de simuladores con alto realismo que permitían que los pilotos practicaran sin riesgo. El resultado fue la disminución de los errores humanos a menos del 30\%. Fue así como el Dr. David Gaba aplicó los conceptos de simulación a la sala de operaciones para mejorar el entrenamiento de los anestesiólogos y así lograr seguridad para el paciente.

En 1960 la empresa Leardal creó el simulador Anne, al cual inició el camino en la simulación, debido a que sus características eran ser simple, económico, práctico y efectivo. Este simulador podía obstruir la vía aérea y con él se podían realizar compresiones torácicas. En 1960 también se desarrolló otro simulador, llamada SimOne, considerado de alta fidelidad ${ }^{3}$.

El desarrollo de la simulación clínica moderna ha sido influenciado por eventos importantes como la protección a los individuos bajo experimentación (en la declaración de Helsinki, 1964), la exigencia de la seguridad del paciente, la calidad de la atención en la educación médica y el desarrollo tecnológico en computación electrónica. Nuevos materiales, háptica y la realidad virtual se encuentran en constante desarrollo.

Paralelo al desarrollo de simuladores sofisticados de tareas específicas quirúrgicas, diagnósticas y de procedimientos se desarrolla la investigación sobre la utilidad de la simulación clínica en el campo del desarrollo de las competencias clínicas ${ }^{4}$.

\section{¿Por qué es importante la simulación?}

\section{Teoría del aprendizaje de adultos}

La andragogía es la ciencia que estudia la educación en los adultos. Se han propuesto diferentes enfoques y principios, la mayoría de ellos basados en los aspectos para mejorar la motivación y proporcionar orientación adecuada. De hecho, hay aspectos de la motivación y reflexión extrínseca que desempeñan un papel central en la educación médica que no se abordan clásicamente por la andragogía; algunas otras teorías, como el aprendizaje transformativo, abordan este aspecto ${ }^{5}$.

La simulación desempeña un papel importante en el modelo de aprendizaje dirigido por el estudiante. Permite crear una «necesidad de saber», ya que imita situaciones reales y brinda al estudiante la oportunidad de practicar procedimientos en un área segura, un entorno controlado. Con esta técnica es posible abordar las enfermedades de mayor prevalencia, superando la variabilidad esperada de escenarios reales en un entorno hospitalario.

Durante la simulación se realiza un aspecto crítico: la retroalimentación constante ${ }^{6}$. Se realiza por medio de informes, que deben considerarse como una oportunidad única para reforzar el aprendizaje del adulto, así como proporcionar motivación externa y estimular la reflexión guiada. Se debe analizar cómo la experiencia afecta la práctica futura para mejorar el rendimiento?.

\section{Simulación en educación médica}

\section{Definición}

La simulación se ha definido como la técnica de imitar el comportamiento de alguna situación o proceso (ya sea médico, económico, militar, mecánico, etc.) por medio de una situación o aparato adecuadamente análogo, especialmente con el propósito de estudiar o capacitar al personal.

\section{Tipos de simuladores}

Existen varias clasificaciones de los diferentes tipos de simuladores que se utilizan en el área médica:

- Simuladores de uso específico, también conocidos como part task trainers. Estos modelos representan una extremidad, parte del cuerpo o estructura. Se utilizan generalmente para ayudar a la adquisición de habilidades técnicas, de procedimiento o psicomotoras, como la punción venosa, la oftalmoscopia y la cateterización. Hay simuladores de tareas parciales más sofisticados, como el Harvey y el Simulator-K, que son sistemas cardiovasculares de alta fidelidad diseñados para ayudar a los estudiantes a reconocer los hallazgos cardiacos auscultatorios comunes $^{8}$.

- Paciente estandarizado. Un paciente estandarizado $(P E)$ es un individuo al que se entrena para representar de forma coherente y precisa una 
enfermedad o problema de salud concreto, con el objetivo de enseñar y evaluar habilidades interpersonales y clínicas. La autenticidad y el feedback son las dos variables principales que determinan la calidad de la actuación y el entrenamiento de esta técnica9 ${ }^{9}$.

- Simuladores virtuales en pantalla. Por medio de programas de computación permiten entrenar, evaluar y tomar decisiones, y hacen posible el trabajo de varios estudiantes al mismo tiempo ${ }^{10}$.

- Simuladores de tareas complejas. Mediante el uso de modelos y software de alta fidelidad se logra una representación tridimensional de un espacio anatómico.

- Simuladores de paciente completo. Son simuladores con software de alta fidelidad que permiten desarroIlar competencias clínicas complejas, así como el trabajo en equipo ${ }^{11}$.

El concepto de fidelidad en los simuladores se ha utilizado para definir el grado de realismo de los modelos y de la experiencia en la que se usan, dividiéndolos en tres niveles:

- Simulación de baja fidelidad. Modelos que simulan solo una parte del organismo, es una buena herramienta para adquirir habilidades motrices en un procedimiento simple o examen físico, por ejemplo, la instalación de una vía venosa periférica y la auscultación cardiaca.

- Simulación de fidelidad intermedia. Se combina el uso de una parte anatómica con programas computacionales de menor complejidad que permiten al instructor manejar variables fisiológicas básicas, con el objetivo de lograr el desarrollo de una competencia. Se pueden utilizar varios medios como el PE, simulación híbrida (se utiliza a un PE con una parte de su cuerpo reemplazada por un entrenador de tareas) y simuladores de háptica (permite sentir las sensaciones mediante el tacto).

- Simulación de alta fidelidad. Implica tener espacios físicos de desempeño profesional que sean muy parecidos a la realidad (quirófano, servicio de urgencias). Se debe contar con todos los elementos reales de trabajo y con un simulador de alta fidelidad.

La calidad de la fidelidad de la simulación depende de la capacidad de los docentes para definir las competencias que desea trabajar por medio del entrenamiento y de la evaluación, así como de la calidad de construcción de escenarios pertinentes, objetivos respecto al grado de complejidad necesaria para el nivel de los estudiantes ${ }^{12}$.

\section{Ventajas educativas del entrenamiento basado en la simulación}

La evidencia científica sugiere que la educación médica basada en simulación supera a la enseñanza tradicional para el aprendizaje de habilidades y destrezas, es una herramienta ideal para los nuevos retos de la educación médica:

- Trabajo en equipo.

- Trabajo multidisciplinario.

- Entrenamiento en situaciones poco comunes.

- Estandarización de programas académicos.

- Práctica libre de riesgos.

- Escenario controlado.

- Repetición de procedimientos en el momento que se decida.

- Las habilidades técnicas adquiridas mediante esta herramienta de la simulación son transferibles a la realidad.

- Aprendizaje por medio del método de prueba y error, que permite al alumno confrontar sus experiencias acumuladas hasta ese momento y lo desafía a una reacción constructivista.

- Permite realizar análisis en tiempo real.

- Los estudiantes perciben la simulación como una experiencia positiva.

Algunas consideraciones de la enseñanza basada en simulación son (Tabla 1):

- El facilitador debe estar en capacitación continua sobre las nuevas herramientas de enseñanza que le permitan hacer uso de los simuladores.

- Los medios técnicos limitan el proceso de enseñanza.

- Se debe considerar que existen situaciones que no se pueden simular, motivo por el cual se deben considerar los objetivos de aprendizaje.

- Un escenario de simulación puede producir una emoción de estrés.

\section{Simulación en medicina de urgencias}

Al igual que en otras áreas de la salud, la simulación en medicina de urgencias se está empezando a explorar. Sus aplicaciones pudieran enfocarse en el desarrollo de habilidades y procedimientos. Algunos ejemplos de esto son la simulación robótica con herramientas didácticas de dramatización, escenografía veraz y posterior análisis de las asistencias simuladas, tras ser grabadas en vídeo, que han supuesto un salto cualitativo importante en la metodología didáctica. Aunque en general se han limitado a técnicas de 
Tabla 1. Generalidades de la simulación

\section{Definición}

Técnica de imitar el comportamiento de alguna situación o proceso (médico, económico, militar, mecánico, etc.) por medio de una situación 0 aparato adecuadamente análogo, especialmente con el propósito de estudiar o capacitar al personal.

\begin{tabular}{|c|c|c|c|c|c|c|}
\hline \multirow{2}{*}{\multicolumn{3}{|c|}{ Etapas de desarrollo de la simulación }} & 1. era & $2^{a}$ & \multicolumn{2}{|l|}{ 3. era } \\
\hline & & & $\begin{array}{l}1929 \\
\text { Simuladores de } \\
\text { vuelo }\end{array}$ & $\begin{array}{l}1960-1980 \\
\text { Desarrollo de } \\
\text { simuladores complejos }\end{array}$ & \multicolumn{2}{|c|}{$\begin{array}{l}\text { Simulación como } \\
\text { complemento }\end{array}$} \\
\hline \multicolumn{2}{|c|}{$\begin{array}{l}\text { La simulación moderna tiene } \\
\text { influencia de } 4 \text { fuerzas: }\end{array}$} & Bioética & $\begin{array}{l}\text { Desarrollo de la } \\
\text { educación médica }\end{array}$ & Seguridad del paciente & \multicolumn{2}{|c|}{ Desarrollo tecnológico } \\
\hline \multicolumn{7}{|c|}{ Clasificación de los simuladores } \\
\hline \multicolumn{2}{|c|}{$\begin{array}{l}\text { Simuladores de uso específico y } \\
\text { baja tecnología }\end{array}$} & $\begin{array}{l}\text { Paciente } \\
\text { estandarizado }\end{array}$ & $\begin{array}{l}\text { Simuladores } \\
\text { virtuales en } \\
\text { pantalla }\end{array}$ & $\begin{array}{l}\text { Simuladores de tareas } \\
\text { complejas }\end{array}$ & \multicolumn{2}{|c|}{$\begin{array}{l}\text { Simuladores de paciente } \\
\text { completo }\end{array}$} \\
\hline \multicolumn{2}{|c|}{$\begin{array}{l}\text { Task trainers, permiten desarrollo } \\
\text { de habilidades psicomotoras }\end{array}$} & $\begin{array}{l}\text { Actores entrenados } \\
\text { para actuar como } \\
\text { paciente }\end{array}$ & $\begin{array}{l}\text { Simula situaciones } \\
\text { en computadora }\end{array}$ & $\begin{array}{l}\text { Modelos de alta } \\
\text { fidelidad, ambiente } \\
\text { virtual }\end{array}$ & \multicolumn{2}{|c|}{$\begin{array}{l}\text { Permite el desarrollo de } \\
\text { tareas complejas }\end{array}$} \\
\hline \multicolumn{7}{|c|}{ Clasificación de los simuladores según su realismo } \\
\hline \multicolumn{2}{|l|}{ Baja fidelidad } & \multicolumn{2}{|l|}{ Fidelidad intermedia } & \multicolumn{3}{|l|}{ Alta fidelidad } \\
\hline \multicolumn{2}{|c|}{$\begin{array}{l}\text { Modelos que simulan una parte } \\
\text { del cuerpo (extremidades) }\end{array}$} & \multicolumn{2}{|c|}{$\begin{array}{l}\text { Es una combinación entre una parte del } \\
\text { cuerpo y un programa computacional }\end{array}$} & \multicolumn{3}{|c|}{$\begin{array}{l}\text { Escenarios clínicos realistas con simuladores de } \\
\text { tamaño real (competencias complejas) }\end{array}$} \\
\hline \multirow{2}{*}{$\begin{array}{l}\text { Tipos de } \\
\text { metodologías de } \\
\text { simulación } \\
\text { (niveles) }\end{array}$} & Nivel 0 & Nivel 1 & Nivel 2 & Nivel 3 & Nivel 4 & Nivel 5 \\
\hline & $\begin{array}{l}\text { Simulaciones } \\
\text { escritas }\end{array}$ & $\begin{array}{l}\text { Simuladores de baja } \\
\text { fidelidad }\end{array}$ & $\begin{array}{l}\text { Simuladores } \\
\text { virtuales }\end{array}$ & Paciente estandarizado & $\begin{array}{l}\text { Simulador } \\
\text { intermedio }\end{array}$ & $\begin{array}{l}\text { Alta } \\
\text { fidelidad }\end{array}$ \\
\hline \multicolumn{7}{|c|}{ Fases de una simulación } \\
\hline $\begin{array}{l}\text { Introducción al } \\
\text { ambiente }\end{array}$ & $\begin{array}{l}\text { Reunión } \\
\text { sobre el } \\
\text { simulador }\end{array}$ & Teoría & $\begin{array}{l}\text { Reunión sobre el } \\
\text { caso }\end{array}$ & Escenario & Debriefing & Finalización \\
\hline Ventajas & \multicolumn{6}{|c|}{$\begin{array}{l}\text { Ambiente controlado y seguro } \\
\text { Permite crear y reproducir situaciones o escenarios a demanda } \\
\text { Permite el entrenamiento sistemático y repetido de habilidades prácticas y competencias } \\
\text { El proceso de aprendizaje se basa en la práctica y la reflexión }\end{array}$} \\
\hline
\end{tabular}

entrenamiento (intubación, control de la vía aérea con dispositivos alternativos, cricotirotomía, drenaje de neumotórax, canalización venosa, desfibrilación, etc. $)^{13}$.

\section{Conclusiones}

Hoy en día no todos los hospitales cuentan con áreas de capacitación mediante educación basada en simulación, motivo por el cual se debe sensibilizar para generar áreas para el desarrollo de habilidades clínicas y destrezas, simulación de alta fidelidad y simulación virtual, para capacitar al personal sanitario formado y en educación continua, con el fin de dar inicio a una nueva etapa en la educación para la salud, creando programas de acuerdo con los recursos y la morbimortalidad nacional, logrando un impacto positivo en la salud, mediante educación sin riesgo y con ello contribuir a la mejora de la atención de los pacientes en las áreas médicas. Específicamente en el área de urgencias, en donde la cantidad de procedimientos invasivos (tanto diagnósticos como terapéuticos) necesarios de dominar son elevados, la simulación resulta un herramienta que considerar a fin de que se puedan desarrollar las habilidades necesarias sin los riesgos potenciales al emplearse en pacientes reales. Sería ideal que los programas académicos no solo la consideren en su currículo, sino que se generen verdaderas líneas de implementación e investigación. 


\section{Financiamiento}

La presente investigación no ha recibido ayudas específicas provenientes de agencias del sector público, sector comercial o entidades sin ánimo de lucro.

\section{Conflicto de intereses}

Los autores declaran no tener conflicto de intereses.

\section{Responsabilidades éticas}

Protección de personas y animales. Los autores declaran que para esta investigación no se han realizado experimentos en seres humanos ni en animales.

Confidencialidad de los datos. Los autores declaran que en este artículo no aparecen datos de pacientes.

Derecho a la privacidad y consentimiento informado. Los autores declaran que en este artículo no aparecen datos de pacientes.

\section{Bibliografía}

1. Maestre J, Palazuelos J, Moral J. La simulación clínica como herramienta para facilitar el cambio de cultura en las organizaciones de salud: aplicación práctica de la teoría avanzada del aprendizaje. Rev Colomb Anestesiol. 2014;42(2):124-8.

2. Swanwick T. Understanding medical education: Evidence, theory and practice. 1st edition. Wiley-Blackwell (John Wiley \& Sons); 2010.

3. Rubio-Martínez R. Pasado, presente y futuro de la simulación en anestesiología. Rev Mex Anest. 2012;35(3);186-91.

4. Stone RJ. Haptic feedback: A brief history from telepresence to virtual reality. En: Brewster S, Murray-Smith R, editores. Haptic human-computer interaction. Haptic $\mathrm{HCl}$ 2000. Lecture Notes in Computer Science. Berlin, Heidelberg: Springe; 2058. pp.1-16.

5. Knowles MS, Holton EF III, Swanson RA. The adult learner: The definitive classic in adult education and human resource development. Routledge; 2014.

6. Abela J. Adult learning theories and medical education: a review. Malta Medical Journal. 2009;21(1):11-8

7. Kaufman DM. Applying educational theory in practice. BMJ 2003;326(7382):213-6

8. Takashina T, Shimizu M, Katayama H. A new cardiology simulator. Cardiology. 1997;88(5):408-13.

9. Ruiz-Moral Roger, Caballero-Martínez Fernando. Programa para seleccionar y entrenar pacientes estandarizados en el contexto de un currículo universitario de simulación clínica. FEM (Ed. impresa) [Internet]. 2014;17(4):199-204. Disponible en: http://scielo.isciii.es/scielo.php?scrip$\mathrm{t}=\mathrm{sci}$ arttext\&pid=S2014-98322014000400005\&lng=es. http://dx.doi. org/10.4321/S2014-98322014000400005.

10. Maran NJ, Glavin RJ. Low- to high-fidelity simulation - a continuum of medical education? Med Educ. 2003;37(Suppl 1):22-8.

11. Lane J, Slavin S, Ziv A. Simulation in medical education: A review. Simul Gaming. 2001;32(3):297-314.

12. Amaya Afanador A. Simulación clínica y aprendizaje emocional. Rev Colomb Psiquiat. 2012:41(Supl. 1):44-51.

13. Ayuso F, Nogué R, Coll Vinent B, Fernández Esáin B, Miró O. Docencia en medicina de urgencias y emergencias. Anales Sis San Navarra. 2010;33(Supl. 1):203-13. 